\title{
Characterisation of hollow Russian doll microspheres
}

\author{
Shakiela Begum • Ian P. Jones · Chengge Jiao • \\ Daniel E. Lynch $\cdot$ Jon A. Preece
}

Received: 22 December 2009/Accepted: 5 April 2010/Published online: 11 May 2010

(C) The Author(s) 2010. This article is published with open access at Springerlink.com

\begin{abstract}
Poly(1-methylpyrrol-2-yl)squaraine (PMPS) particles have been characterised using SEM. The PMPS particles were used as templates to prepare bare silica and iron-silica hollow spheres, which were characterised using TEM and SEM. The PMPS particles and the hollow spheres are not uniformly sized and are agglomerated. The hollow spheres with larger diameters $(>900 \mathrm{~nm})$ contain an internal 'Russian doll' structure. The iron-silica hollow spheres are fused to one another, and the hollow spheres have a heterogeneous wall thickness. The silica and ironsilica hollow spheres both aggregate by size. There are two different size populations (for the diameter) of the bare
\end{abstract}

S. Begum · J. A. Preece $(\bowtie)$

School of Chemistry, University of Birmingham, Edgbaston,

Birmingham B15 2TT, UK

e-mail: j.a.preece@bham.ac.uk

S. Begum

e-mail: sxb398@bham.ac.uk

I. P. Jones

School of Metallurgy and Materials, University of Birmingham,

Edgbaston, Birmingham B15 2TT, UK

e-mail: i.p.jones@bham.ac.uk

C. Jiao

FEI Company, Focussed Ion Beam Laboratory, Bristol, UK

e-mail: chengge.jiao@uk.feico.com

D. E. Lynch $(\bowtie)$

Exilica Limited, The Technocentre, Puma Way,

Coventry CV1 2TT, UK

e-mail: d.lynch@exilica.co.uk silica and iron-silica hollow spheres. The smaller silica spheres have thinner walls compared to the larger silica hollow spheres. The larger silica hollow spheres and the iron-silica hollow spheres have similar wall thicknesses. The iron compound in the iron-silica hollow spheres has an oxidation state of $3+$ and is crystalline.

\section{Introduction}

In recent years, there has been a great deal of interest in the fabrication of uniformly sized hollow microspheres, as they can be used to encapsulate inorganic or organic materials and for the manufacture of advanced materials $[1,2]$. The sacrificial core process is one of the most frequently used techniques to produce hollow inorganic spheres [1-12]. The sacrificial core approach involves the use of a solid organic or inorganic spherical particle as the template. The core-shell structure is produced by coating the core with inorganic precursors or nanoparticles which are crosslinked to form the shell. Hollow spherical particles are subsequently generated by removing the template from the core-shell structure thermally $[1-10]$ or chemically $[1-3$, $11,12]$.

Hollow Russian doll microspheres (which consist of two or more shells) are produced using similar routes used to prepare single wall hollow spheres, except the template is not a solid particle. The template is spherical and has a multilayered internal structure. The multilayered template walls are coated with a layer of silica precursors (on both sides) which are polymerised to form the silica shells [1316]. Removal of the template yields hollow Russian doll microspheres.

Hollow sphere dimensions and morphology are characterised using electron microscopy (i.e. SEM or TEM 
Fig. 1 Preparation of a bare silica hollow spheres and b iron-silica hollow spheres using PMPS particles

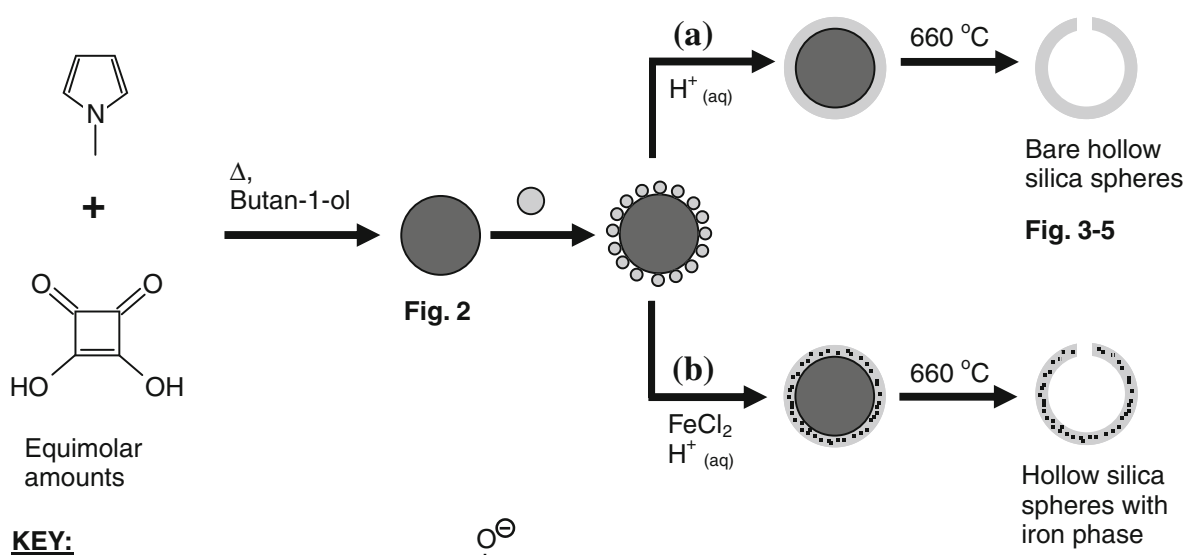

KEY: microsphere of

Fig. 8-12 images). The chemical composition is determined using energy dispersive X-ray spectrometry (EDX) or electron energy loss spectrometry (EELS) in the TEM. EELS is used to probe the chemical composition and band structure of bulk materials [17] or at interfaces [18]. EELS has been used to characterise $d$-block metals, i.e. iron $(\mathrm{Fe})$ [19-24], and light elements, i.e. silicon (Si) [25] and oxygen (O) [26], all of which are significant here. Chemical shifts and intensity variations of edges in the EELS spectra are used to deduce valence information about iron and its oxides.

Lynch et al. [8] described the preparation of hollow silica spheres as shown in Fig. 1. In this study, sacrificial templates used were spherical particles of poly(1-methylpyrrol-2-yl)squaraine (PMPS), whose average diameter was given as $1,300 \mathrm{~nm}$. Removal of these particles resulted in hollow shells of $50 \%$ the template diameter so the resultant shells had an average size of $650 \mathrm{~nm}$. In the present study, more detailed investigations of the physical characteristics of PMPS particles, the hollow silica shells made from the PMPS particles and new hollow iron oxide/ silica composite shells (also made from PMPS particles) are undertaken.

The average diameter of the PMPS particles has been determined using SEM. The diameters (TEM and SEM) and wall thicknesses (SEM) have been determined for the hollow spheres of bare silica and iron-silica. The chemical composition (EDX) of the iron-silica hollow spheres has been verified, and EELS has been used to determine the oxidation state of the iron compound. Selected area diffraction patterns (DPs) have been used to show that the iron compound in the hollow sphere walls is crystalline.

\section{Experimental}

Materials

Chemicals used to prepare the PMPS template and hollow spheres were purchased from Sigma-Aldrich. The chemicals and materials used for SEM and TEM experiments were purchased from Agar Scientific. All the chemicals and materials were used as-received.

Preparation of silica and iron-silica hollow spheres

These hollow spheres were prepared using the same procedure as described by Lynch et al. [8], except that during the acid hydrolysis stage, the silicated particles were suspended in a solution of iron(II) chloride $(1.00 \mathrm{~g}, 3.7 \mathrm{mmol})$ in $\mathrm{HCl}$ (30 $\mathrm{mL}, 0.1 \mathrm{M})$ to prepare the iron-silica hollow spheres.

SEM characterisation of the microstructure

\section{Preparation of the SEM specimen}

The powdered specimen was supported on a stub using double-sided carbon tape, and the specimen was coated with gold using a SC7640 sputter coater or carbon using an Edwards 306 coating unit.

\section{Scanning electron microscopes}

The following scanning electron microscopes were used: (i) JEOL JSM-6060 LV and (ii) Philips XL30 ESEM-FEG. The microscopes were calibrated by obtaining a micrograph of a calibration grid using the same parameters used 
to obtain the specimen micrograph. The size of the scale bar of the specimen micrograph was checked using the features on the calibration grid micrograph.

\section{Focussed ion beam (FIB) SEM characterisation of the iron-silica particles}

The FIB cross-sectional micrographs were acquired using a FEI Quanta 200 3D Dual Beam microscope. Double-sided carbon tape was used to hold the sample on the stub.

TEM characterisation of the microstructure

\section{Preparation of the specimen block: addition of specimen to resin}

Araldite resin was chosen as the embedding medium because it polymerizes uniformly with very little shrinkage. Therefore, the embedded hollow spheres were not altered during the polymerisation of the resin. In addition, Araldite resin shows very good stability under the electron beam. The Araldite resin was formulated from Araldite CY212 (3 mL), EPON substitute Agar 100 RESIN (5 mL), DDSA (hardener, $13 \mathrm{~mL}$ ) and DBP (plasticiser, $0.6 \mathrm{~mL}$ ) via stirring for $5 \mathrm{~min}$. The liquid accelerator (DMP 30, $0.5 \mathrm{~mL}$ ) was added to the mixture while stirring resulting in a colour change from yellow to orange. The mixture was placed under vacuum $\left(20 \mathrm{~min}, 23^{\circ} \mathrm{C}\right)$. Two beam capsules $(20 \mathrm{~mm} \times 9 \mathrm{~mm})$ were completely filled using a syringe $(5 \mathrm{~mL})$ containing the air free resin. The powdered specimen $(\sim 0.5-1 \mathrm{mg})$ was added to each resin-filled beam capsule, and the mixture inside the capsule was gently agitated to help the hollow spheres sink into the resin. The beam capsules were heated $\left(60{ }^{\circ} \mathrm{C}\right)$ under vacuum (30 min). The resin was left to cure $\left(60{ }^{\circ} \mathrm{C}\right)$ at ambient pressure overnight to yield a polymerised resin-specimen block.

\section{Preparation of ultra thin sections}

The Araldite specimen blocks were trimmed by hand using a double-sided razor blade until the cutting face was pyramidal shaped and the hollow spheres were exposed and within the trapezoid-shaped pyramid top. The block was secured into a REICHERT-JUNG ultramicrotome, and was fine trimmed using a glass knife set at a clearance angle of $4^{\circ}$. The glass ultramicrotome knife was prepared by breaking a glass strip ( $1 \mathrm{~cm}$ thick) using a knife maker (type 7801A). Ultrathin (80-100 nm) sections of the block were obtained using a diamond knife set at a clearance angle of $6^{\circ}$. The gold coloured sections were captured onto formvar-coated grids by sweeping the section out of the water.

\section{Transmission electron microscopes}

The TEM micrographs were obtained using Philips CM20 TEM $(\mathrm{kV}=200)$ and Jeol 1200 TEM $(\mathrm{kV}=80)$. FEI Tecnai F20 Field Emission TEM equipped with an Oxford Instruments EDX spectrometer and a Gatan EELS spectrometer was used to obtain: (i) EDX microanalysis, (ii) EELS spectra and (iii) images of the $\mathrm{Fe}$-silica hollow spheres.

\section{EDX line analysis}

An ultra thin $(80-100 \mathrm{~nm})$ Araldite section through the $\mathrm{Fe}-$ silica hollow spheres mounted on a formvar-coated copper grid was coated with carbon (as described in the "Experimental" section). A line (resolution: fine) was drawn on the STEM bright field image of the Fe-silica hollow sphere using the Oxford Instruments Link ISIS program. Spectra of the elements $\mathrm{Fe}, \mathrm{Si}$ and $\mathrm{O}$ were acquired for $100 \mathrm{~s}$ on the selected area.

\section{Preparation of EELS samples}

The metallic iron sample used as an EELS standard here was prepared by a dual beam FIB (of Ga ions) and supplied by Dr. G West from IPTME at the University of Loughborough. The $\gamma-\mathrm{Fe}_{2} \mathrm{O}_{3}$ or FeS EELS standard was prepared by smearing a carbon-coated grid over dry ground powder, and then lightly shaking off the excess powder. One sample of the iron-silica hollow spheres (supplied by Exilica) was also prepared this way for EELS analysis.

\section{EELS experimental}

The powdered standards or iron-silica hollow spheres were analysed by selecting a thin edge of a standard particle or hollow sphere fragment. The ultramicrotome section of the iron-silica hollow spheres on the formvar-coated $\mathrm{Cu}$ grid was carbon coated by thermal evaporation. Each spectrum was acquired using a dispersion of $0.2 \mathrm{eV}$ and a spectrometer entrance aperture of $2 \mathrm{~mm}$. The drift tube voltage was set at $650 \mathrm{eV}$ and an integration time of $10 \mathrm{~s}$ with 5-10 readouts per acquisition was used. After acquisition, the energy scale was calibrated and the background was removed using a power law fit to $I=A E^{-r}$ (where $I=$ intensity, $E=$ energy and $A$ and $r=$ fitting parameters). The spectrum was deconvoluted using the Fourier gain method.

\section{Selected area electron diffraction (SAED) pattern}

The sample analysed was an ultramicrotome section through $\mathrm{Fe}$-silica hollow spheres embedded in Araldite 
resin supported on a formvar-coated $\mathrm{Cu}$ grid. An electron DP was obtained from particles of the iron compound, embedded in the wall of a hollow sphere, using the Philips CM20 TEM $(200 \mathrm{kV})$ in SAED mode. The area of interest was selected using an SAED aperture of $10 \mu \mathrm{m}$, and the diameter of the selected specimen area was $\sim 400 \mathrm{~nm}$.

\section{Results and discussion}

\section{Characterisation of the template}

The synthesis scheme is shown in Fig. 1, which details the figures from the "Results and discussion" section. The fine powder nature of the washed and dried PMPS particles is illustrated in Fig. 2a. An SEM micrograph of the goldcoated (1-2 nm) PMPS particles is shown in Fig. 2b, c, which is a histogram of calibrated frequency versus diameter $(\mathrm{nm})$. The average diameter is $1924 \pm 45 \mathrm{~nm}$, $n=116$ (Table 1 entry i). The $1,924 \mathrm{~nm}$ average diameter
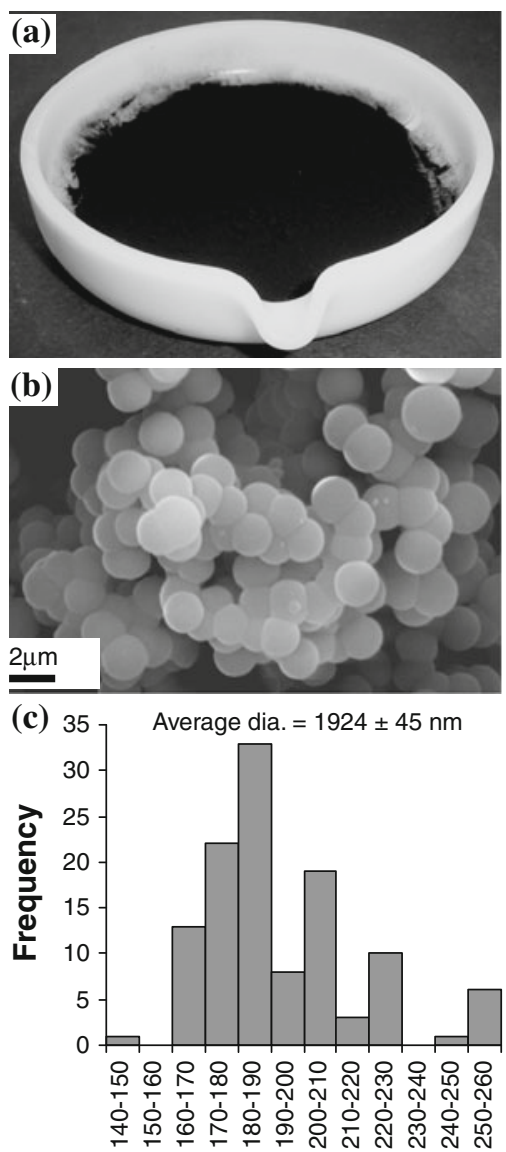

Diameter $\times 10(\mathrm{~nm})$

Fig. 2 a Photograph of PMPS particles, b JEOL 6060 SEM micrograph of gold-coated PMPS particles, $\mathbf{c}$ histogram of calibrated frequency versus diameter $(\mathrm{nm})$ for gold-coated PMPS particles in (b) for the PMPS particles studied in this paper is larger than the diameter $(1,300 \mathrm{~nm})$ reported previously [8].

Characterisation of bare silica hollow spheres

\section{SEM assessment of silica hollow sphere diameter}

A FEG ESEM micrograph of the carbon-coated silica hollow spheres is shown in Fig. 3a, b. Gold-coated silica hollow spheres were examined using FEG ESEM and Jeol 6060 SEM, micrographs from both microscopes showed that the spheres have an uneven appearance, small bumps were seen on the surface of the spheres. Therefore, carbon coating was used as an alternative to gold coating to remove artefacts from the image. Statistical analysis on this area (Fig. 3a), shown in the histogram (Fig. 3c; Table 1 entry ii), reveals that the diameter is $937 \pm 11 \mathrm{~nm}$ $(n=97)$. This diameter is corroborated by a second analysis (Table 1 entry iii) acquired using Jeol 6060 SEM micrographs of gold-coated silica hollow spheres where the diameter is $905 \pm 22 \mathrm{~nm}(n=73)$. It should be noted that the TEM analysis (Table 1 entry iv) shows a much smaller diameter $(650 \pm 47 \mathrm{~nm})$, but this is a small sample size $(n=5)$. The diameter of the hollow silica spheres is significantly smaller than the average PMPS bead diameter [8]. A number of examples are found in the literature [3-7] where calcination of the template (polystyrene bead) causes a $<10 \%$ shrinkage of shell diameter. The calcination procedures used in other studies are not significantly different from the procedure used by Lynch et al. [8]. The PMPS beads have been shown to shrink in diameter with increasing temperature [8], because the PMPS beads degrade differently to polystyrene beads the two processes are not directly comparable. For example, when polystyrene is used as the template [3-7], a surfactant is also used to adhere the silica precursor to the template, whereas Lynch et al. [8] did not use any surfactant to adhere the TEOS to the PMPS template. Further investigations into the synthetic process are required to work out why silica shell diameter shrinks significantly when PMPS beads are used.

TEM assessment of silica hollow sphere diameter and wall thickness using bright field (BF) TEM micrographs

In order to determine the thickness of the hollow sphere walls, ultra thin $(\sim 80-100 \mathrm{~nm})$ sections through silica hollow spheres embedded in Araldite resin were prepared. Side views of the sectioning process are illustrated in Fig. 4a, highlighting that hollow spheres 1 and 2 will overestimate the thickness, whilst hollow sphere 3 reveals the correct thickness. The sections were placed on formvarcoated copper grids, and then examined using the TEM. 
Table 1 Summary of size characterisation results

\begin{tabular}{|c|c|c|c|c|c|c|c|c|c|}
\hline Entry & Material & $\begin{array}{l}\text { Outer diameter } \\
(\mathrm{nm})\end{array}$ & $\begin{array}{l}\text { Inner diameter } \\
(\mathrm{nm})\end{array}$ & $\begin{array}{l}\text { Wall thickness } \\
(\mathrm{nm})\end{array}$ & $\begin{array}{l}\text { Diameter: wall } \\
\text { thickness }\end{array}$ & $\begin{array}{l}\text { Sample size } \\
\text { (n) }\end{array}$ & Coating & $\begin{array}{l}\text { Electron } \\
\text { microscope }\end{array}$ & Figure \\
\hline $\mathrm{i}$ & $\mathrm{PMPS}^{\mathrm{a}}$ & $1924 \pm 45$ & - & - & - & 116 & $\mathrm{Au}$ & $\mathrm{b}$ & $2 b$ \\
\hline ii & Silica & $937 \pm 11$ & - & - & - & 97 & $\mathrm{C}$ & $\mathrm{c}$ & $3 a$ \\
\hline iii & & $905 \pm 22$ & - & - & - & 73 & $\mathrm{Au}$ & $\mathrm{b}$ & - \\
\hline iv & & $650 \pm 47$ & $505 \pm 42$ & $73 \pm 8$ & 8.9 & 5 & - & d & $4 b$ \\
\hline $\mathrm{v}$ & & $1081 \pm 59$ & $848 \pm 67$ & $116 \pm 14$ & 9.3 & 8 & - & d & 5 \\
\hline vi & Iron-silica & $1421 \pm 43$ & - & - & - & 158 & $\mathrm{C}$ & $\mathrm{c}$ & $8 a$ \\
\hline vii & & $1480 \pm 54$ & - & - & - & 92 & $\mathrm{Au}$ & $\mathrm{b}$ & - \\
\hline viii & & $1309 \pm 32$ & $1079 \pm 32$ & $115 \pm 5$ & 11.4 & 20 & - & e & $9 a$ \\
\hline ix & & $1370 \pm 69$ & $1128 \pm 65$ & $121 \pm 8$ & 11.3 & 33 & - & d & - \\
\hline $\mathrm{x}$ & & $2724 \pm 249$ & $2493 \pm 225$ & $116 \pm 31$ & 23.5 & 3 & - & d & $9 b$ \\
\hline
\end{tabular}

a The diameter shown here is a representative value, but these templates were not the batch used to template the silica and iron-silica hollow spheres

b Jeol 6060 SEM

c Philips XL-30 FEG ESEM

d Jeol 1200 EX TEM

e Philips CM20 TEM
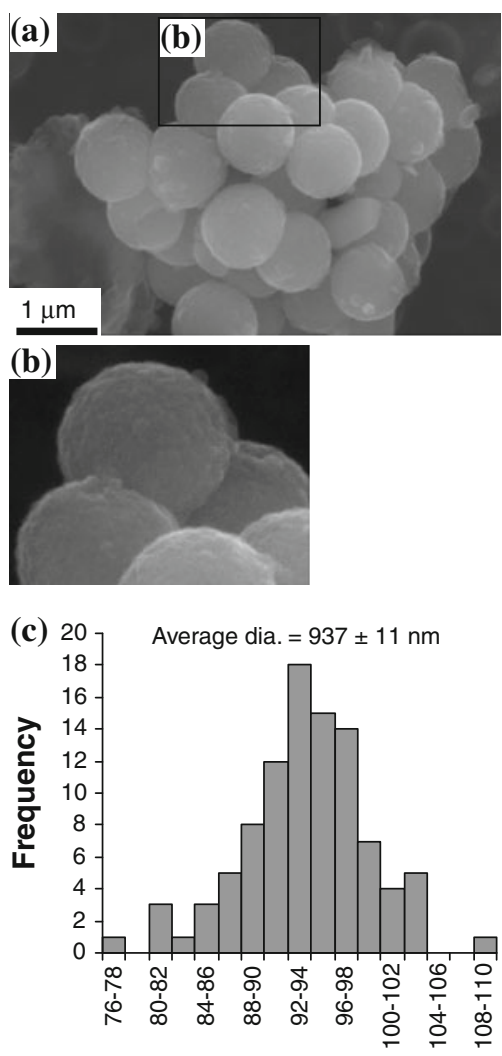

Diameter x 10 (nm)

Fig. 3 a FEG ESEM micrograph of carbon-coated bare silica hollow spheres, b enlarged area of (a) showing the surface smoothness, $\mathbf{c}$ histogram of calibrated frequency versus diameter $(\mathrm{nm})$ for carboncoated silica hollow spheres in (a) (a)
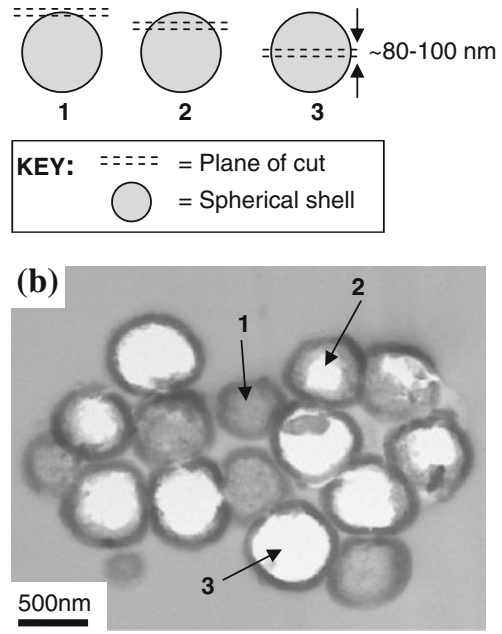

Fig. 4 a Illustration of different positions where the hollow spheres can be sectioned, b JEOL 1200 TEM micrograph of an ultra thin section through silica hollow spheres showing examples of (a)

Figure $4 \mathrm{~b}$ is a bright field TEM micrograph of an ultra thin section through bare silica hollow spheres embedded in Araldite resin. The dark grey coloured circles (hollow sphere 1) are the hollow spheres where the diamond knife just skimmed over the top (Fig. 4a). The silica hollow spheres with thicker walls (hollow sphere 2) are the hollow spheres sectioned closer to the top/bottom (Fig. 4a). Hollow sphere 3 is white in the centre due to the dissimilar electron density contrast between the surrounding resin and 
the hollow sphere interior: this proves the hollow spheres are hollow and do not fill up with resin. Only the hollow spheres with thinner walls were used to estimate the average wall thickness and sphere diameter (this procedure limited the number of hollow spheres that could be included in the statistical analysis, $n=5$ ). Table 1 (entry iv) shows the average diameters and wall thickness measured using the TEM micrograph (Fig. 4b).

Figure 5 is a second bright field TEM micrograph of an ultra thin section through bare silica hollow spheres. This TEM image (Fig. 5) reveals a new feature of internal hollow spherical structures ('Russian doll'-like effect).

We propose one hypothesis that the internal structure of the hollow spheres forms because of the suspected porosity of the PMPS particles, thus a small percentage have silica monomer absorbed deep enough within the particle such that it can form its own separate shell inside the outer shell (as shown in Fig. 6). However, there could be other mechanisms. In addition, some of the hollow spheres are co-joined, presumably by formation of the silica shell over two fused PMPS templates (as shown in Fig. 7).

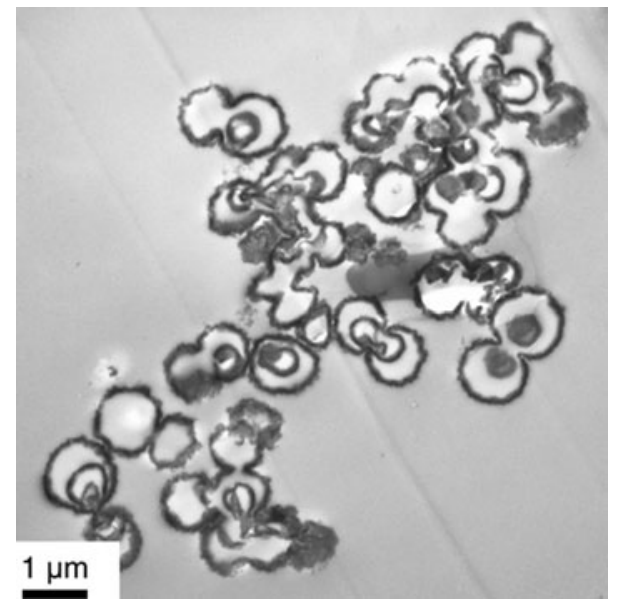

Fig. 5 Philips CM20 TEM of micrograph of an ultra thin section of silica hollow spheres embedded in Araldite resin
The outer diameter (Table 1, entry v) measured from Fig. $5(1081 \pm 59 \mathrm{~nm}, n=8)$ is closer to the diameters measured using the SEM micrographs $(937 \pm 11 \mathrm{~nm}$, $n=97$; Table 1, entry ii). The walls of the hollow spheres in Fig. 5 are thicker $(116 \pm 14 \mathrm{~nm}, n=8)$ compared to Fig. 4 ( $73 \pm 8 \mathrm{~nm}, n=5)$. Although the larger hollow spheres have greater wall thickness, the ratio of the diameter:wall thickness does not change significantly with particle size (Table 1, entries iv-v). This indicates that a fixed amount of silica adheres to the PMPS particles.

What is clear from entries ii-v of Table 1 is that there is much greater variation in the dispersity of the silica hollow spheres than previously reported [8]. In addition, the SEM and TEM micrographs reveal that hollow spheres of roughly the same size aggregate together.

\section{Characterisation of silica hollow spheres with iron}

The iron-silica composite hollow spheres produced using PMPS particles (Fig. 1) have not been characterised previously, and the characterisation is reported for the first time in the following sections.

\section{SEM assessment of iron-silica hollow sphere diameter}

SEM micrographs were obtained for carbon-coated (Fig. 8; Table 1, entry vi) and gold-coated (Figure not shown, Table 1, entry vii) iron-silica hollow spheres. In both cases, the surface of the hollow spheres did not appear smooth. The average diameters for carbon- and gold coated iron-silica hollow spheres agreed within experimental error $[1421 \pm 43 \mathrm{~nm}(n=158)$ and $1480 \pm 54 \mathrm{~nm}(n=$ 92), respectively].

\section{TEM assessment of iron-silica hollow sphere diameter} and wall thickness using BF micrographs

Ultra thin $(\sim 80-100 \mathrm{~nm})$ sections of iron-silica hollow spheres embedded in Araldite resin were placed on
Fig. 6 Schematic representation of how the internal structure may form inside the hollow spheres

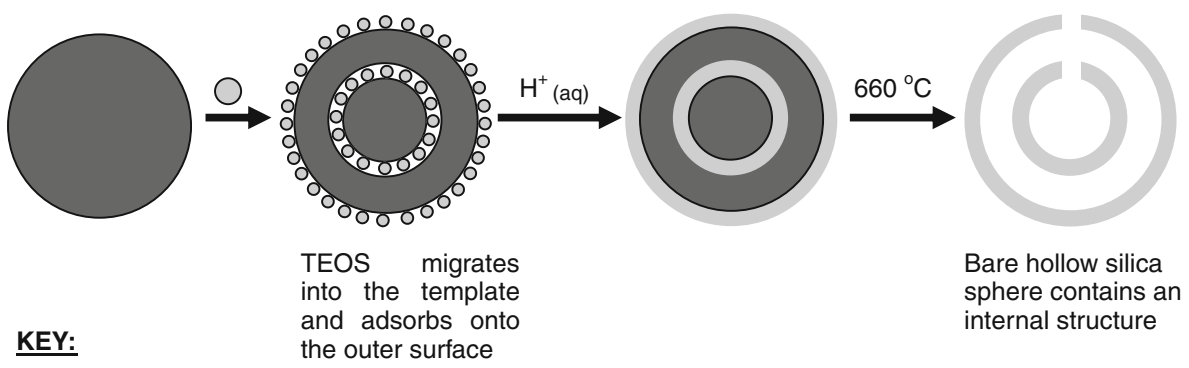

$=$ PMPS particle
$O=$ TEOS precursors 
Fig. 7 Illustration of how fused hollow spheres might form

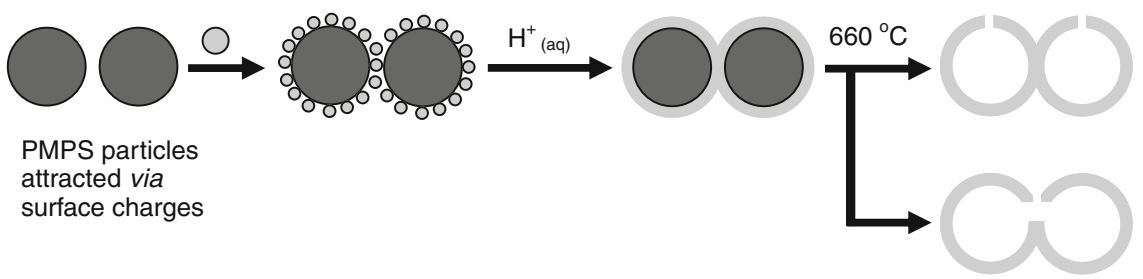

(a)

Fused bare hollow silica spheres
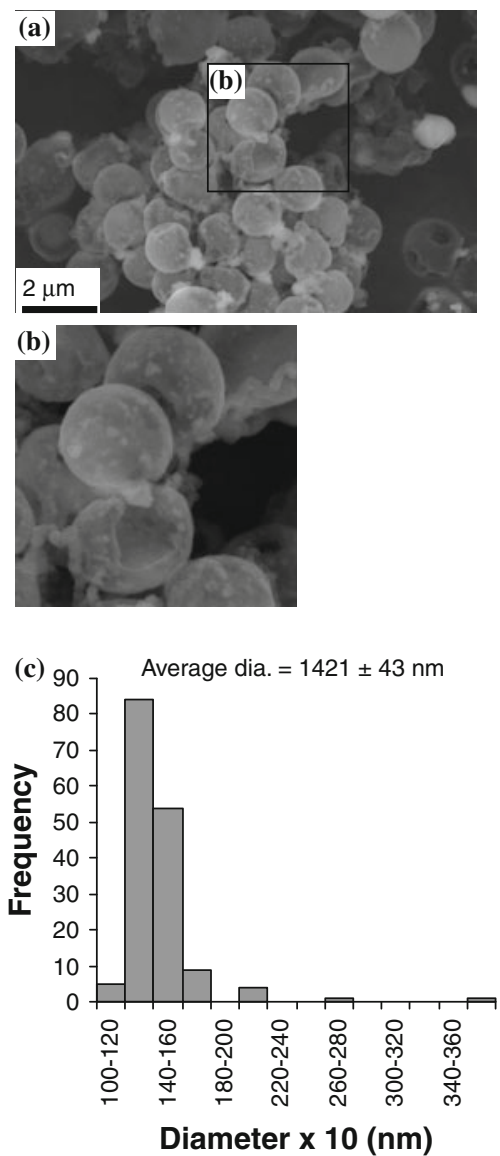

Fig. 8 a FEG ESEM micrograph of carbon-coated iron-silica hollow spheres, b enlarged area of (a) showing the surface smoothness, c histogram of calibrated frequency versus diameter $(\mathrm{nm})$ for carboncoated iron-silica hollow spheres in (a)

formvar-coated copper grids, and then examined using the TEM. These images (Fig. 9a, b) show that the iron-silica hollow spheres are hollow, and contain an internal 'Russian doll' structure like the bare silica hollow spheres (Fig. 5). The Fe-silica diameters (Table 1, entries vi-x) are larger than those recorded for the silica hollow spheres (Table 1, entries ii-v). However, we are not suggesting that the ironsilica hollow spheres are statistically larger than the silica hollow spheres, as the sample sizes are too small to make this differentiation.
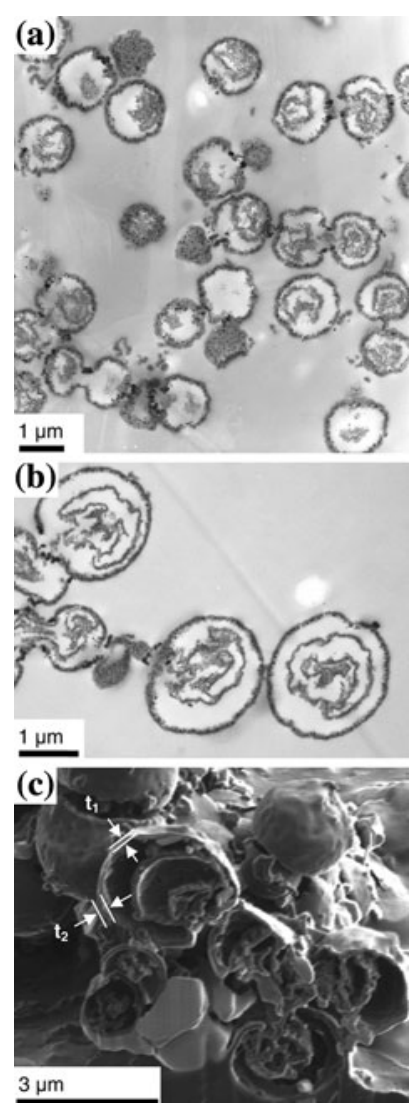

Fig. 9 a Philips CM20 TEM micrograph of an ultra thin section of iron-silica hollow spheres, b JEOL 1200 TEM micrograph of an ultra thin section of the larger iron-silica hollow spheres, c FEI Quanta 200 3D Dual Beam microscope FIB cross section micrograph for ironsilica hollow spheres

Table 1 (entry viii) also shows the average diameter and wall thickness measured using the TEM micrograph (Fig. 9a). The average outer diameter $(1309 \pm 32 \mathrm{~nm}$, $n=20)$ is similar to the diameters that were measured from the SEM micrograph $(1421 \pm 43 \mathrm{~nm}, n=158$, Fig. 8).

Figure $9 \mathrm{~b}$ is a TEM micrograph of an ultra thin section of the larger iron-silica hollow spheres. This micrograph shows that the larger spheres that were observed using the SEM also contain a hollow internal structure, like the smaller spheres in Fig. 9a. The larger spheres (Fig. 9b) 
have similar wall thickness $(116 \pm 31 \mathrm{~nm}$, Table 1 entry $\mathrm{x})$ to that of the smaller spheres $(115 \pm 5 \mathrm{~nm}$, Table 1 entry viii). The diameter:wall thickness ratios of these results indicate that the maximum hollow sphere thickness achieved through this process of preparing hollow spheres is $121 \pm 8 \mathrm{~nm}$; the thickness of the iron-silica hollow sphere does not depend on the diameter of the PMPS particle template. This rule may also apply to the bare silica hollow spheres as they are also formed using a similar process, but we did not observe particles of $2,000 \mathrm{~nm}$ diameter to check this hypothesis.

The BF TEM micrographs (Fig. 9) of the iron-silica hollow spheres show that the hollow spheres are made up of at least two phases, as revealed by the difference in contrasts of the grey scale in the wall. The lighter grey background of the hollow sphere wall predominated, interspersed with dark grey points of another phase, presumably containing the iron (see later section on EDX analysis).

\section{FIB characterisation of iron-silica hollow spheres}

The FIB cross-sectional micrographs (Fig. 9c) were acquired using a FEI Quanta 200 3D Dual Beam microscope. The two key points are (i) the internal structures are predominantly hollow spheres and (ii) the wall thicknesses are variable around each hollow sphere. For example, the hollow sphere thickness for $t_{1}$ is $58 \mathrm{~nm}$, whereas $t_{2}$ is $116 \mathrm{~nm}$.

\section{EDX line analysis of an iron-silica hollow sphere}

An EDX line scan was conducted on an iron-silica hollow sphere in order to determine the concentrations of the different elements ( $\mathrm{Fe}, \mathrm{Si}$ and $\mathrm{O}$ ) in the hollow sphere walls. Figure 10a shows a BF STEM image of a carboncoated ultra thin $(80-100 \mathrm{~nm})$ section through iron-silica hollow spheres acquired using the FEI Tecnai F20 Field Emission TEM. Figure 10b is an enlarged area of Fig. 10a showing superimposed EDX line scans of $\mathrm{Fe}, \mathrm{Si}$ and $\mathrm{O}$. The line analysis shows the predominant phase in the hollow sphere is silica and the highest concentration of the silica is in the outer hollow sphere wall. The concentrations of the oxygen and iron are similar in the outer hollow sphere wall and internal structure.

\section{EELS analysis of an iron-silica hollow sphere}

Electron energy loss spectrometry (EELS) point analysis was conducted in order to determine the oxidation state of the iron phase in the iron-silica hollow spheres. The EELS measurements were initially conducted on iron valence standards $\left(\mathrm{Fe}^{(0)}, \mathrm{Fe}^{(\mathrm{II})}, \mathrm{Fe}^{(\mathrm{III})}\right)$. The EELS spectra for the standards are shown in Fig. 11a. The spectra of the standards display a similar chemical shift pattern to that reported in the literature $[19,22,23]$, i.e. the energy of $\mathrm{Fe}^{(0)}<\mathrm{Fe}^{(\mathrm{II})}<\mathrm{Fe}^{(\mathrm{III})}$. The $L_{2,3}$ edge shapes seen in Fig. 11a are similar to those seen in the literature, and the height of the $L_{2}$ increases in the following order: $\mathrm{Fe}^{(0)}>\mathrm{Fe}^{(\mathrm{II})}>\mathrm{Fe}^{(\mathrm{III})}$.

Two samples of the iron-silica hollow spheres were prepared differently. The first sample (A) was a carboncoated ultramicrotome section ( 80-100 nm thick) on a formvar-coated $\mathrm{Cu}$ grid. The second sample (B) was prepared by crushing the hollow spheres and smearing the crushed powder on the TEM grid. The ultramicrotome section had a uniform thickness and therefore any area of the sample could be used, whereas for the crushed powder, only particle edges were selected to reduce plural scattering. The spectra from samples $\mathrm{A}$ and $\mathrm{B}$ are shown in Fig. 11a, b, respectively. The $L_{2,3}$ edge shapes and positions of the EELS spectra acquired from the powdered sample and ultrathin section are both similar to the $\gamma-\mathrm{Fe}_{2} \mathrm{O}_{3}$
Fig. 10 FEI Tecnai F20 Field Emission STEM bright field micrograph of a carbon-coated ultra thin $(80-100 \mathrm{~nm})$ section through iron-silica hollow spheres embedded in Araldite resin (b) enlarged area of (a) showing superimposed EDX line scans for $\mathrm{Fe}, \mathrm{Si}$ and $\mathrm{O}$
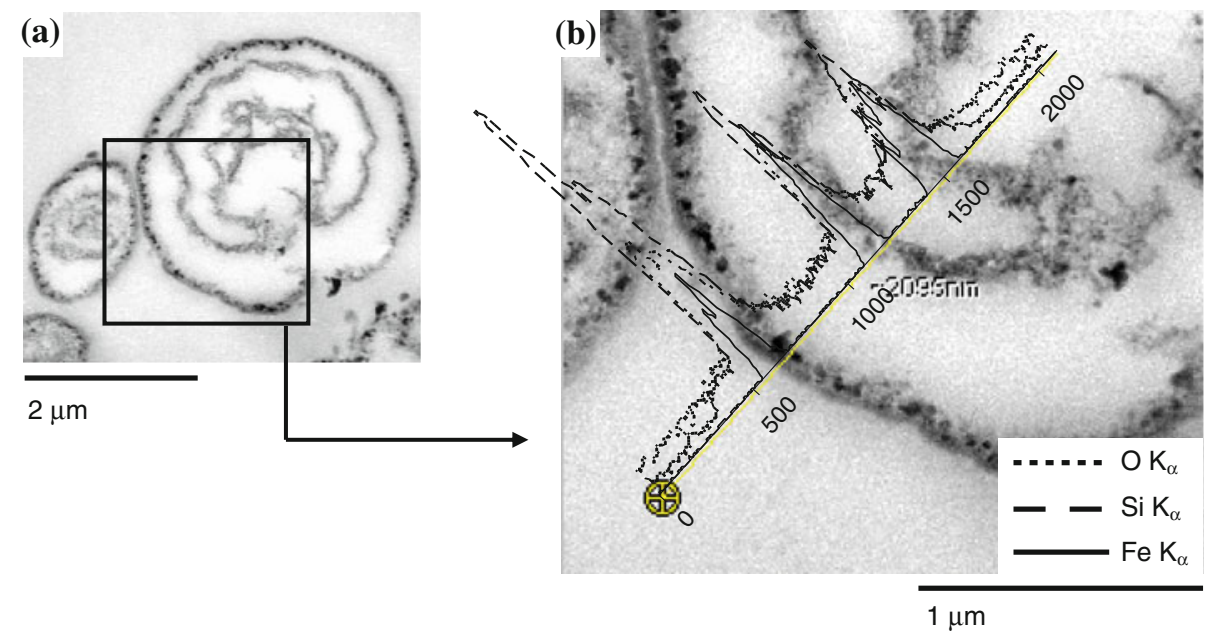

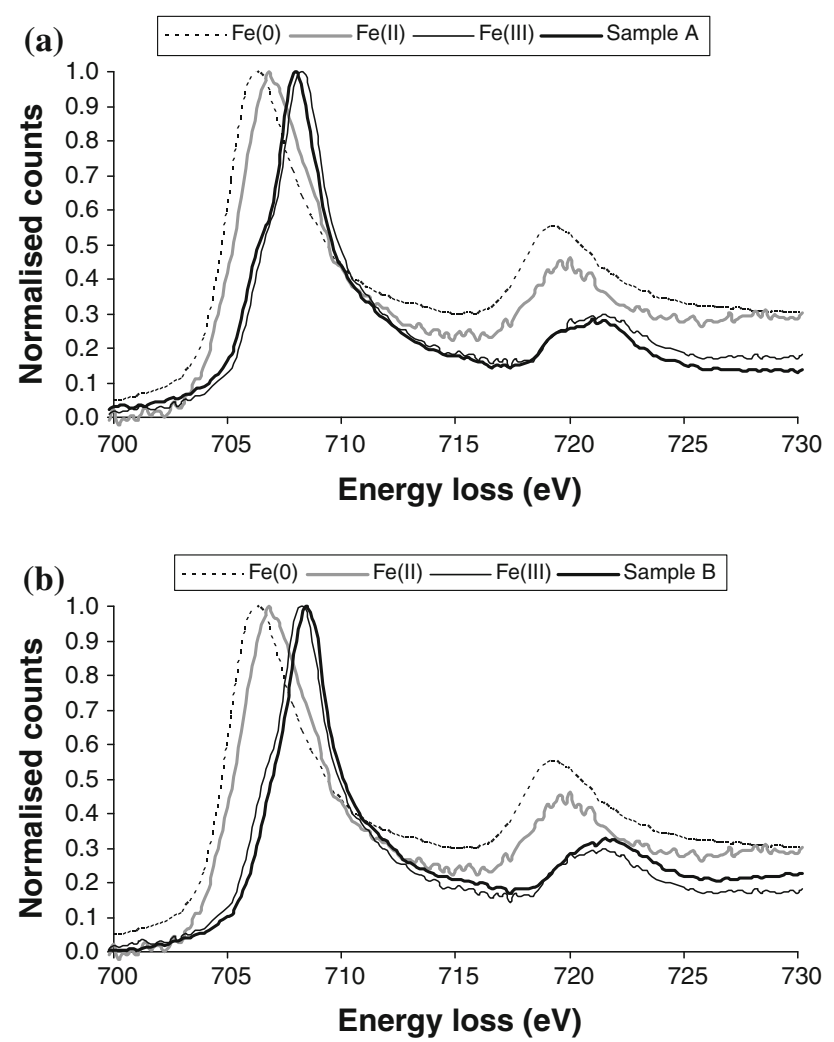

Fig. 11 EELS spectra from a sample A (ultramicrotome section through iron-silica hollow spheres), and b sample B (crushed ironsilica hollow spheres dispersed on a carbon-coated $\mathrm{Cu}$ grid) superimposed on EELS spectra of the $\mathrm{Fe}^{(0)}, \mathrm{Fe}^{(\mathrm{II})}$ and $\mathrm{Fe}^{(\mathrm{III})}$ standards

standard $[22,23]$. Therefore, it is likely that the oxidation state of the iron compound is $3+$.

The results from the EELS spectra are summarised in Table 2. The powdered and sectioned samples both have similar chemical shifts. The electron energy loss near edge structure of the sectioned sample is better resolved compared to the crushed hollow spheres: this could be due to thickness effects. The white line intensity ratios (WLR) have also been summarised in Table 2 . The WLR $\left(L_{3} / L_{2}\right)$ was calculated using the maximum intensity method [27]. The WLR of the standards obey the trend $\mathrm{Fe}^{(0)}<$ $\mathrm{Fe}^{(\mathrm{II})}<\mathrm{Fe}^{(\mathrm{III})}$. The WLR of iron-silica is similar to the WLR of the $\mathrm{Fe}^{(\mathrm{III})}$ standard.

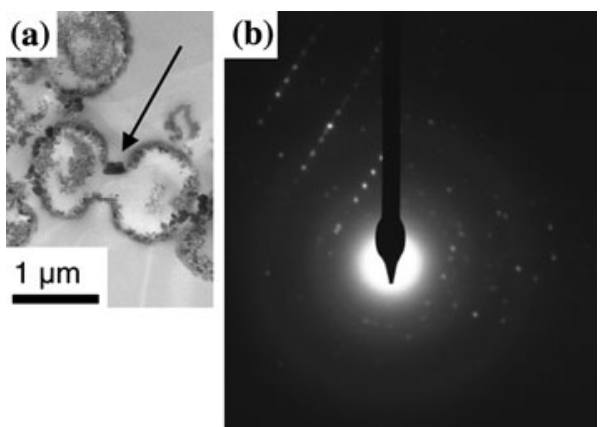

Fig. 12 a BF TEM image of an ultramicrotome section through an iron-silica hollow sphere embedded in Araldite resin (arrow indicates the area analysed using SAED), b SAED pattern of an iron-silica hollow sphere

Selected area electron diffraction (SAED) of an iron-silica hollow sphere

The DP was obtained by placing the electron beam on an iron crystal in the hollow sphere wall (indicated by an arrow in Fig. 12a). Figure 12b shows the DP for the ironsilica hollow sphere wall. This result shows that the iron phase in the hollow spheres is crystalline and not amorphous. The small $\mathbf{g}$-vector is close to the smallest expected for $\gamma-\mathrm{Fe}_{2} \mathrm{O}_{3}$ [28]. The silica phase of the iron-silica hollow spheres was found to be amorphous.

\section{Conclusion}

Poly(1-methylpyrrol-2-yl)squaraine (PMPS) particles were characterised using SEM. The PMPS particles appear to be agglomerated and the particle surface has an even appearance. The silica and iron-silica hollow spheres were characterised using TEM and SEM. The results showed that the surface of the silica hollow spheres has a relatively even appearance and the spheres are hollow. The larger silica hollow spheres (diameter $>900 \mathrm{~nm}$ ) contain an interior structure. It is not clear why the interior structure appears only in hollow spheres with diameters over 900 $\mathrm{nm}$. The iron-silica spheres are also hollow and contain an interior structure and the iron-silica hollow sphere surface does not have an even appearance. The results obtained

Table 2 Summary of EELS results

\begin{tabular}{llllllll}
\hline Sample type & Sample & Oxidation state & $L_{3}(\mathrm{eV})$ & $L_{2}(\mathrm{eV})$ & $L_{3} / L_{2}$ & Sample preparation & Sample thickness $(\mathrm{nm})$ \\
\hline Standards & $\mathrm{Fe}$ & 0 & 706.6 & 719.4 & 1.8 & FIBed & $80-100$ \\
& $\mathrm{FeS}$ & $2+$ & 706.8 & 719.6 & 2.2 & Powder & 100 \\
& $\gamma-\mathrm{Fe}_{2} \mathrm{O}_{3}$ & $3+$ & 708.2 & 721.4 & 3.4 & Powder & $70-100$ \\
Exilica sample & Iron-silica & & 708.4 & 721.6 & 3.1 & Powder & $80-100$ \\
& & & 708.0 & 720.8 & 3.7 & Section & \\
\hline
\end{tabular}


using the FIB microscope agreed with the results obtained using the TEM. FIB micrographs also showed that the iron-silica hollow spheres are physically connected to one another, and some of the hollow spheres have a heterogeneous wall thickness. The average diameter of the PMPS particles $(1924 \mathrm{~nm})$ studied in this paper was found to be larger than the average diameter $(1300 \mathrm{~nm})$ of the particles studied by Lynch et al. [8]. The silica and iron-silica hollow spheres both aggregate by size. There are two different size populations for the bare silica and iron-silica hollow spheres, therefore different groupings showed different average diameters. The smaller silica spheres have thinner walls compared to the larger silica hollow spheres. The larger silica hollow spheres and the iron-silica hollow spheres have similar wall thicknesses. EDX showed that iron is present in the hollow sphere wall, and the structure inside the hollow sphere. EELS analysis has been used to show that the iron phase in iron-silica hollow spheres has an oxidation state of $3+$ and it is likely that the iron phase is $\mathrm{Fe}_{2} \mathrm{O}_{3}$. Diffraction patterns suggest that the ironcontaining phase in the hollow spheres is crystalline and likely to be $\gamma-\mathrm{Fe}_{2} \mathrm{O}_{3}$.

Acknowledgements This work was supported by EPSRC, The University of Birmingham, AWM and ERDF through science city. The PMPS, hollow sphere specimens were supplied by Exilica Limited, and the Fe (0) EELS standard was supplied by Dr G West from IPTME at the University of Loughborough.

Open Access This article is distributed under the terms of the Creative Commons Attribution Noncommercial License which permits any noncommercial use, distribution, and reproduction in any medium, provided the original author(s) and source are credited.
5. Ding XF, Yu KF, Jiang YQ, Hari B, Zhang HB, Wang ZC (2004) Mater Lett 58:3618

6. Chen YW, Kang ET, Neoh KG, Greiner A (2005) Adv Funct Mater 15:113

7. Zhang L, Acunzi MD, Kappl M et al (2009) Langmuir 25:2711

8. Lynch DE, Nawaz Y, Bostrom T (2005) Langmuir 21:6572

9. He L, Jia Y, Meng F, Li M, Liu J (2009) J Mater Sci 44:4326. doi: 10.1007/s10853-009-3645-y

10. Shiho H, Kawahashi N (2000) J Colloid Interface Sci 226:91

11. Li ZZ, Wen LX, Shao L, Chen JF (2004) J Controlled Release 98:245

12. Wang X, Chen Y, Zhou W, Huang Z, Guo Z, Hu Y (2009) J Mater Sci 44:4710. doi:10.1007/s10853-009-3728-9

13. Yang M, Ma J, Zhang C, Yang Z, Lu Y (2005) Angew Chem Int Ed 44:6727

14. Jung JH, Ono Y, Sakurai K, Sano M, Shinkai S (2000) J Am Chem Soc 122:8648

15. Zhou GW, Chen YJ, Yang JH, Yang SH (2007) J Mater Chem $17: 2839$

16. Li J, Ding S, Zhang C, Yang Z (2009) Polymer 50:3943

17. Ahn CC (2004) Transmission electron energy loss spectrometry in materials science and the EELS ATLAS. Wiley-VCH, Weinheim

18. Hu KX, Jones IP (2005) J Phys D Appl Phys 38:183

19. Leapman RD, Grunes LA, Fejes PL (1982) Phys Rev B 26:614

20. Wang F, Malac M, Egerton RF (2007) Micron 38:371

21. Jasinski J, Pinkerton KE, Kennedy IM, Leppert VJ (2005) Sens Actuators B 109:19

22. Paterson JH, Krivanek OL (1990) Ultramicroscopy 32:319

23. Colliex C, Manoubi T, Ortiz C (1991) Phys Rev B 44:11402

24. Chen S-Y, Gloter A, Zobelli A, Wang L, Chen C-H, Colliex C (2009) Phys Rev B 79:1

25. Ito Y, Winkler D, Jain H, Williams DB (1997) J Non-Cryst Solids 222:83

26. Keast VJ, Scott AJ, Brydson R, Williams DB, Bruley J (2001) J Microsc 203:135

27. Riedl T, Gemming T, Wetzig K (2006) Ultramicroscopy 106:284

28. Pearson WB (1967) A handbook of lattice spacings and structures of metals and alloys. Pergamon, Oxford

\section{References}

1. Caruso F (2000) Chem Eur J 6:413

2. Liu J, Liu F, Gao K, Wu J, Xue D (2009) J Mater Chem 19:6073

3. Lu Y, McLellan J, Xia YN (2004) Langmuir 20:3464

4. Zhang G, Yu Y, Chen X et al (2003) J Colloid Interface Sci 263:467 